\title{
Idiopathic Renal Infarction and Anticoagulation
}

\author{
Maurice I. Khayat ${ }^{1} \quad$ Robert Nee $^{1}$ Dustin J. Little ${ }^{1}$ \\ ${ }^{1}$ Department of Nephrology, Walter Reed National Military Medical \\ Center, Bethesda, Maryland, United States \\ TH Open 2019;3:e331-e334.
}

\author{
Stephen W. Olson ${ }^{1}$
}

\section{Introduction}

Idiopathic renal infarction (iRI) is rare and the pathophysiology is not well understood. ${ }^{1}$ There is no consensus treatment strategy for iRI, because previous studies have not reported long-term outcomes based on therapeutic intervention. ${ }^{2-15}$ We sought to determine if anticoagulation or nonanticoagulation was associated with a higher incidence of recurrent arterial thrombosis, de novo venous thrombosis, bleeding event, or development of long-term hypertension, proteinuria, or chronic kidney disease (CKD).

\section{Methods}

We conducted a retrospective iRI cohort study to compare long-term outcomes between anticoagulation and nonanticoagulation therapy. We first queried the military electronic medical record (EMR) system, composed of approximately 10 million current and prior service members and their dependents, for the International Classification of Diseases 9 and 10 codes for renal infarction (593.81 and N28.0, respectively). There were 689 potential cases of renal infarction identified. After a full review of the EMR, only 322 were found to have radiologic confirmation of renal infarction. A total of 103 cases were determined to be idiopathic renal infarcts as defined by the absence of known thromboembolic risk factors, hypercoagulability, or vascular pathology etiologies (-Fig. 1). A thromboembolic risk factor was defined by diagnostic evidence or documentation by a cardiologist of a patent foramen ovale, ventricular dilatation or aneurysm, valvular vegetations, atrial fibrillation, or significant aorta plaque burden $(n=103)$. Hypercoagulability was defined by documentation by a hematologist or presence of factor V Leiden, protein C or S deficiency, anti-B2-glycoprotein antibody, anticardiolipin antibody, antiphospholipid antibody, lupus anticoagulant antibody, genetic risk, systemic lupus erythematosus, systemic vasculitis, malignancy, inflammatory bowel disease, pregnancy, or nephrotic syndrome on chart review $(n=44)$. Renal vascular pathology was defined by documentation in a vascular surgery note or

received

July 23, 2019

accepted

August 27, 2019
DOI https://doi.org/

10.1055/s-0039-1698757. ISSN 2512-9465.
Address for correspondence Stephen W. Olson, MD, Department of Nephrology, Walter Reed National Military Medical Center, 8901 Rockville Pike, Bethesda, MD 20889, United States

(e-mail: Stephen.w.olson.mil@mail.mil).

evidence of renal artery stenosis or aneurysm, trauma, or association with abdominal or vascular surgery in close anatomic proximity on chart review $(n=58)$. Fourteen cases did not have adequate documentation of a negative secondary work-up for iRI assignment. Prespecified background clinical data ( - Table 1 ) and primary outcomes were then collected for all 103 iRI cases. Long-term outcomes included recurrent arterial thrombosis by imaging, de novo venous thrombosis by imaging, new or worsening hypertension (two blood pressure readings of $>140 / 90 \mathrm{~mm} \mathrm{Hg}$ or the addition/escalation of antihypertension mediations), new proteinuria ( $\geq 1+$ on urinalysis in the absence of infection), a bleeding event (confirmed by specialist or emergency department with imaging or procedure), or new stage 3 CKD (estimated glomerular filtration rate less than $60 \mathrm{~mL} / \mathrm{min} / 1.73 \mathrm{~m}^{2}$ by CKD-Epi Creatinine equation) after iRI diagnosis. Initial comparison groups were anticoagulation $(n=47)$ and nonanticoagulation treatment. The nonanticoagulation group $(n=56)$ was further subdivided into an antiplatelet group $(n=37)$ and a group with either no treatment or undocumented over-the-counter aspirin (ASA) treatment $(n=19)$. Univariate analyses were performed with Chi-square testing for categorical variables (Fisher's exact test used for violations of Cochran's assumptions) and Wilcoxon rank-sum test for continuous variables with nonparametric distribution.

\section{Results}

The median follow-up period after iRI was 4.3 years and the background clinical characteristics were similar between treatment groups (-Table 1). The overall rates of recurrent arterial and de novo venous thrombosis were $1 \%(1 / 103)$ and $2 \%(2 / 103)$ respectively and there was no significant difference between treatment groups (-Table 2 ). The anticoagulation group had a higher rate of bleeding event than the nonanticoagulation group and the antiplatelet subgroup ( 13 vs. $0 \%, p=0.008$ and 13 vs. $0 \%, p=0.03$, respectively). Incident proteinuria occurred at iRI in $8 \%$ of cases $(8 / 103)$, $88 \%(7 / 8)$ of which resolved during follow-up. Resolution

\footnotetext{
(c) 2019 Georg Thieme Verlag KG Stuttgart · New York
}

License terms

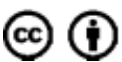




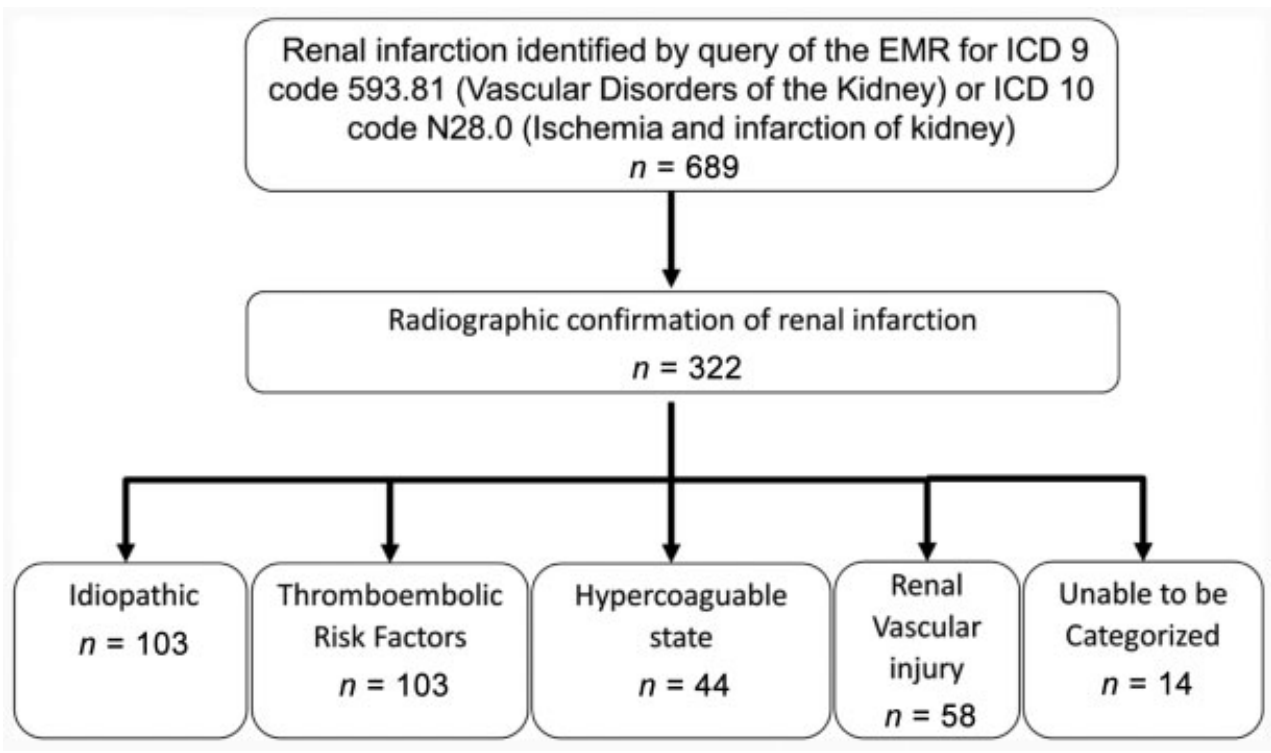

Fig. 1 A flow diagram of idiopathic renal infarction case selection.

Table 1 Baseline characteristics of cases before renal infarction overall and divided into treatment groups

\begin{tabular}{|c|c|c|c|c|}
\hline & $\begin{array}{l}\text { All cases } \\
(n=103)\end{array}$ & $\begin{array}{l}\text { Anticoagulation } \\
(n=47)\end{array}$ & $\begin{array}{l}\text { Nonanticoagulation } \\
(n=56)\end{array}$ & $\begin{array}{l}\text { Antiplatelet } \\
(n=37)\end{array}$ \\
\hline Age $(y)$ & $\begin{array}{l}42 \\
(36,49)\end{array}$ & $\begin{array}{l}41 \\
(36,49)\end{array}$ & $\begin{array}{l}44 \\
(36,48)\end{array}$ & $\begin{array}{l}46 \\
(39,50)\end{array}$ \\
\hline \multicolumn{5}{|l|}{ Race } \\
\hline White & $46 \%(47 / 103)$ & $45 \%(21 / 47)$ & $46 \%(26 / 56)$ & $49 \%(18 / 37)$ \\
\hline Black & $7 \%(8 / 103)$ & $13 \%(6 / 47)$ & $4 \%(2 / 56)$ & $3 \%(1 / 37)$ \\
\hline Asian & $4 \%(4 / 103)$ & $4 \%(2 / 56)$ & $4 \%(2 / 56)$ & $0 \%(0 / 37)$ \\
\hline Other & $27 \%(28 / 103)$ & $21 \%(10 / 47)$ & $32(18 / 56)$ & $32 \%(12 / 37)$ \\
\hline Unknown & $16 \%(16 / 103)$ & $17 \%(8 / 47)$ & $14 \%(8 / 56)$ & $16 \%(6 / 37)$ \\
\hline Sex (\% male) & $\begin{array}{l}80 \\
(82 / 103)\end{array}$ & $\begin{array}{l}77 \\
(36 / 47)\end{array}$ & $\begin{array}{l}82 \\
(46 / 56)\end{array}$ & $\begin{array}{l}86 \\
(32 / 37)\end{array}$ \\
\hline HTN (\%; baseline) & $\begin{array}{l}27 \\
(28 / 103)\end{array}$ & $\begin{array}{l}23 \\
(11 / 47)\end{array}$ & $\begin{array}{l}30 \\
(17 / 56)\end{array}$ & $\begin{array}{l}38 \\
(14 / 37)\end{array}$ \\
\hline $\begin{array}{l}\text { New/worsening HTN } \\
\text { (\% at iRI diagnosis) }\end{array}$ & $\begin{array}{l}49 \\
(50 / 103)\end{array}$ & $\begin{array}{l}53 \\
(25 / 47)\end{array}$ & $\begin{array}{l}45 \\
(25 / 56)\end{array}$ & $\begin{array}{l}51 \\
(19 / 37)\end{array}$ \\
\hline DM (\% baseline) & $\begin{array}{l}11 \\
(11 / 103)\end{array}$ & $\begin{array}{l}6 \\
(3 / 47)\end{array}$ & $\begin{array}{l}14 \\
(8 / 56)\end{array}$ & $\begin{array}{l}16 \\
(6 / 37)\end{array}$ \\
\hline \multicolumn{5}{|l|}{ AKI (\%at iRI diagnosis) } \\
\hline SCr $25 \%$ rise & $9(8 / 92)$ & $7(3 / 41)$ & $10(5 / 51)$ & $6(2 / 34)$ \\
\hline SCr $50 \%$ rise & $1(1 / 92)$ & $2(1 / 41)$ & $0(0 / 51)$ & $0(0 / 34)$ \\
\hline $\begin{array}{l}\text { Stage } 3 \text { CKD (\% eGFR } \leq 60 \mathrm{~mL} / \mathrm{min} / 1.73 \mathrm{~m}^{2} \\
\text { at iRI diagnosis) }\end{array}$ & $\begin{array}{l}0 \\
(0 / 92)\end{array}$ & $\begin{array}{l}0 \\
(0 / 40)\end{array}$ & $\begin{array}{l}0 \\
0 \\
(0 / 52)\end{array}$ & $\begin{array}{l}0 \\
(0 / 27)\end{array}$ \\
\hline $\begin{array}{l}\text { Proteinuria } \geq 1+ \\
\text { (baseline) }\end{array}$ & $\begin{array}{l}1 \\
(1 / 73)\end{array}$ & $\begin{array}{l}33 \\
(1 / 30)\end{array}$ & $\begin{array}{l}0 \\
(0 / 43)\end{array}$ & $\begin{array}{l}0 \\
(0 / 27)\end{array}$ \\
\hline $\begin{array}{l}\text { New Proteinuria } \geq 1+ \\
\text { (\% at iRI diagnosis) }\end{array}$ & $\begin{array}{l}9 \\
(8 / 93)\end{array}$ & $\begin{array}{l}17 \\
(7 / 42)\end{array}$ & $\begin{array}{l}2^{\mathrm{a}} \\
(1 / 51)\end{array}$ & $\begin{array}{l}0^{\mathrm{b}} \\
(0 / 35)\end{array}$ \\
\hline $\begin{array}{l}\text { NSAID use } \\
\text { (\% in year prior to iRI) }\end{array}$ & $\begin{array}{l}77 \\
(79 / 103)\end{array}$ & $\begin{array}{l}85 \\
(40 / 47)\end{array}$ & $\begin{array}{l}70 \\
(39 / 56)\end{array}$ & $\begin{array}{l}70 \\
(26 / 37)\end{array}$ \\
\hline $\begin{array}{l}\text { Long-term followup } \\
\text { (median months, IQR) }\end{array}$ & $\begin{array}{l}51.5 \\
(27,99)\end{array}$ & $\begin{array}{l}48 \\
(22,89)\end{array}$ & $\begin{array}{l}51.5 \\
(29,99)\end{array}$ & $\begin{array}{l}65 \\
(29,114)\end{array}$ \\
\hline
\end{tabular}

Abbreviations: AKI, acute kidney injury; CKD, chronic kidney disease; DM, diabetes mellitus; eGFR, estimated glomerular filtration rate; HTN, hypertension; IQR, interquartile range; iRI, Idiopathic renal infarction; NSAID, nonsteroidal anti-inflammatory drug.

Note: The antiplatelet group is a subgroup of the nonanticoagulation group with clear documentation of treatment.

${ }^{\mathrm{a}} \mathrm{p}=0.02$.

${ }^{\mathrm{b}} \mathrm{p}=0.01$. 
Table 2 A comparison of long-term outcomes after idiopathic renal infarction between anticoagulation and nonanticoagulation groups (top) and between anticoagulation and antiplatelet groups (bottom)

\begin{tabular}{|c|c|c|c|}
\hline & $\begin{array}{l}\text { Anticoagulation } \\
(n=47)\end{array}$ & $\begin{array}{l}\text { Nonanticoagulation } \\
(n=56)\end{array}$ & $p$-Value \\
\hline Recurrent arterial thrombosis (\% cases) & $\begin{array}{l}2 \\
(1 / 47)\end{array}$ & $\begin{array}{l}0 \\
(0 / 56)\end{array}$ & 0.46 \\
\hline De novo venous thrombosis (\%) & $\begin{array}{l}44 \\
(2 / 47)\end{array}$ & $\begin{array}{l}0 \\
(0 / 56)\end{array}$ & 0.21 \\
\hline Bleeding event (\%) & $\begin{array}{l}13 \\
(6 / 47)\end{array}$ & $\begin{array}{l}0 \\
(0 / 56)\end{array}$ & 0.008 \\
\hline Resolution of acute hypertension after iRI (\%) & $\begin{array}{l}16 \\
(4 / 25)\end{array}$ & $\begin{array}{l}20 \\
(5 / 25)\end{array}$ & 1.0 \\
\hline AKI recovery $(\%)$ & $\begin{array}{l}100 \\
(3 / 3)\end{array}$ & $\begin{array}{l}60 \\
(3 / 5)\end{array}$ & 0.46 \\
\hline $\begin{array}{l}\text { Stage } 3 \text { CKD } \\
\text { (\% eGFR } \leq 60 \mathrm{~mL} / \mathrm{min} / 1.73 \mathrm{~m}^{2} \text { follow-up) }\end{array}$ & $\begin{array}{l}0 \\
(0 / 30)\end{array}$ & $\begin{array}{l}0 \\
(0 / 45)\end{array}$ & 1.0 \\
\hline $\begin{array}{l}\mathrm{SCr}>50 \% \text { baseline } \\
\text { (\% last follow-up) }\end{array}$ & $\begin{array}{l}0 \\
(0 / 39)\end{array}$ & $\begin{array}{l}2 \\
(1 / 45)\end{array}$ & 1.0 \\
\hline Resolution of proteinuria (\% negative on UA) & $\begin{array}{l}86 \\
(6 / 7)\end{array}$ & $\begin{array}{l}100 \\
(1 / 1)\end{array}$ & 1.0 \\
\hline \multirow[t]{2}{*}{$\begin{array}{l}\text { Proteinuria } \geq+1 \text { on UA } \\
\text { (\% follow-up) }\end{array}$} & $\begin{array}{l}6 \\
(2 / 34)\end{array}$ & $\begin{array}{l}0 \\
(0 / 46)\end{array}$ & 0.18 \\
\hline & $\begin{array}{l}\text { Anticoagulation } \\
(n=47)\end{array}$ & $\begin{array}{l}\text { Antiplatelet } \\
(n=37)\end{array}$ & $p$-Value \\
\hline Recurrent arterial thrombosis (\% cases) & $\begin{array}{l}2 \\
(1 / 47)\end{array}$ & $\begin{array}{l}0 \\
(0 / 37)\end{array}$ & 1.0 \\
\hline Recurrent venous thrombosis (\%) & $\begin{array}{l}4 \\
(2 / 47)\end{array}$ & $\begin{array}{l}0 \\
(0 / 37)\end{array}$ & 0.50 \\
\hline Bleeding event (\%) & $\begin{array}{l}13 \\
(6 / 47)\end{array}$ & $\begin{array}{l}0 \\
(0 / 37)\end{array}$ & 0.03 \\
\hline Resolution of acute hypertension after iRI (\%) & $\begin{array}{l}16 \\
(4 / 25)\end{array}$ & $\begin{array}{l}26 \\
(5 / 19)\end{array}$ & 0.47 \\
\hline AKI recovery (\%) & $\begin{array}{l}100 \\
(3 / 3)\end{array}$ & $\begin{array}{l}50 \\
(1 / 2)\end{array}$ & 0.40 \\
\hline $\begin{array}{l}\text { Stage III CKD (\%) } \\
\left(\% \text { eGFR } \leq 60 \mathrm{~mL} / \mathrm{min} / 1.73 \mathrm{~m}^{2} \text { last follow-up) }\right.\end{array}$ & $\begin{array}{l}0 \\
(0 / 39)\end{array}$ & $\begin{array}{l}0 \\
(0 / 29) \\
\end{array}$ & 1.0 \\
\hline $\begin{array}{l}\mathrm{SCr}>50 \% \text { baseline } \\
\text { (\% last follow-up) }\end{array}$ & $\begin{array}{l}0 \\
(1 / 39)\end{array}$ & $\begin{array}{l}3 \\
(1 / 29)\end{array}$ & 0.43 \\
\hline Resolution of proteinuria (\% negative on UA) & $\begin{array}{l}86 \\
(6 / 7)\end{array}$ & $\begin{array}{l}\text { NA } \\
(0 / 0)\end{array}$ & 1.0 \\
\hline $\begin{array}{l}\text { Proteinuria } \geq+1 \text { on UA } \\
\text { (\% last follow-up) }\end{array}$ & $\begin{array}{l}6 \\
(2 / 34)\end{array}$ & $\begin{array}{l}0 \\
(0 / 30)\end{array}$ & 0.49 \\
\hline
\end{tabular}

Abbreviations: AKI, acute kidney injury; CKD, chronic kidney disease; eGFR, estimated glomerular filtration rate; iRI, Idiopathic renal infarction; UA, urine analysis.

Note: The anticoagulation group included treatment with warfarin, lovenox, rivaroxaban, and apixaban, and the antiplatelet group included aspirin (ASA) and clopidogrel in addition to ASA in one case.

was also noted in $75 \%(6 / 8)$ patients who presented with acute kidney injury (AKI). Nearly half (50/103) of iRI patients presented with new or worsening hypertension, which resolved in only $18 \%(9 / 50)$ cases. There was no significant difference between treatment groups for longterm resolution of hypertension, proteinuria, or AKI (-Table 2). No iRI cases $(0 / 84)$ had achieved stage 3 CKD and only $2 \%(2 / 80)$ had $\geq 1+$ proteinuria on urinalysis at the last follow-up.

\section{Discussion}

This is the largest retrospective cohort study with the longest follow-up that focuses solely on iRI and the first to compare long-term outcomes between anticoagulation and nonanticoagulation therapy. There was no significant difference in the incidence of recurrent arterial thrombosis or de novo venous thrombosis between treatment groups over 4.3 years median follow-up. In addition there was no 
significant difference in long-term hypertension, proteinuria, or stage 3 CKD following iRI between treatment groups. There was an increased risk of bleeding events in the anticoagulation group. Most previous studies described renal infarction of all causes. ${ }^{2-15}$ All but three studies included less than $10 \mathrm{iRI}$ cases. Oh et al reported a large Asian renal infarction cohort of all etiologies which included 132 iRI cases. ${ }^{2}$ Background clinical characteristics and rate of recurrent iRI (2.3\%) were similar to our cohort. But the median follow-up for iRI was only 12 months and it did not compare long-term outcomes between anticoagulation and nonanticoagulation treatment groups. Bourgault et al also described a cohort of all types of renal infarction which included 27 iRI cases. ${ }^{3}$ Background clinical characteristics were again similar to our cohort but follow-up was limited to 9 months with no evaluation of therapeutic impact on long-term outcomes. Kwon et al compared subgroups with and without AKI and CKD from a cohort of 105 renal infarction cases which included 25 iRI patients. ${ }^{4}$ But, there was no separate iRI subgroup analysis.

This study has all the limitations inherent to a retrospective cohort study. There is some ascertainment bias because not all patients had long-term follow-up data for proteinuria, serum creatinine, or blood pressure. While the negative laboratory and imaging studies for secondary causes were directly reviewed for a majority of iRI cases, a minority of cases only had documentation from subspecialists (cardiologists, hematologists, and nephrologists) outside of the military medical system scanned into our EMR which stated that the secondary causes had been ruled out but without all of the specific results. It is possible that there secondary work-up was erroneously incomplete. Unappreciated causes of renal infarction, such as paroxysmal atrial fibrillation, could have confounded our iRI cases. In addition, multivariate analysis for risk of bleeding event was not possible due to the low event rate relative to the number of covariates.

Overall, our data suggest that nonanticoagulation treatment is as efficacious as anticoagulation therapy for prevention of recurrent or de novo thrombosis after iRI, but with less risk of bleeding. In addition, the data suggest that iRI cases should be monitored closely for optimal long-term blood pressure management. A follow-up prospective study would be required to confirm these findings but will be difficult to execute because of disease rarity, case presentation in emergency room departments, and diverse subspecialty follow-up patterns.

\section{Disclaimer}

The views expressed in this manuscript are those of the authors and do not reflect the official policy of the Department of the Army, Department of Defense, or the U.S. government.

\section{Conflict of Interest}

None declared.

\section{References}

1 Väkevä A, Meri S, Lehto T, Laurila P. Activation of the terminal complement cascade in renal infarction. Kidney Int 1995;47(03): 918-926

2 Oh YK, Yang CW, Kim YL, et al. Clinical characteristics and outcomes of renal infarction. Am J Kidney Dis 2016;67(02):243-250

3 Bourgault M, Grimbert P, Verret C, et al. Acute renal infarction: a case series. Clin J Am Soc Nephrol 2013;8(03):392-398

4 Kwon JH, Oh BJ, Ha SO, Kim DY, Do HH. Renal complications in patients with renal infarction: prevalence and risk factors. Kidney Blood Press Res 2016;41(06):865-872

5 Domanovits H, Paulis M, Nikfardjam M, et al. Acute renal infarction. Clinical characteristics of 17 patients. Medicine (Baltimore) 1999;78(06):386-394

6 Bae EJ, Hwang K, Jang HN, et al. A retrospective study of short- and long-term effects on renal function after acute renal infarction. Ren Fail 2014;36(09):1385-1389

7 Bolderman R, Oyen R, Verrijcken A, Knockaert D, Vanderschueren S. Idiopathic renal infarction. Am J Med 2006;119(04):356. e9-356.e12

8 Faucon AL, Bobrie G, Jannot AS, et al. Cause of renal infarction: a retrospective analysis of 186 consecutive cases. J Hypertens 2018; 36(03):634-640

9 Huang CC, Chen WL, Chen JH, Wu YL, Shiao CJ. Clinical characteristics of renal infarction in an Asian population. Ann Acad Med Singapore 2008;37(05):416-420

10 Caravaca-Fontán F, Pampa Saico S, Elías Triviño S, et al. Acute renal infarction: clinical characteristics and prognostic factors. Nefrologia 2016;36(02):141-148

11 Mesiano P, Rollino C, Beltrame G, et al. Acute renal infarction: a single center experience. J Nephrol 2017;30(01):103-107

12 Antopolsky M, Simanovsky N, Stalnikowicz R, Salameh S, Hiller N. Renal infarction in the ED: 10-year experience and review of the literature. Am J Emerg Med 2012;30(07):1055-1060

13 Lin WL, Seak CJ, Wu JY, Weng YM, Chen HC. Risk factors for development of chronic kidney disease following renal infarction: retrospective evaluation of emergency room patients from a single center. PLoS One 2014;9(06):e98880

14 Silverberg D, Menes T, Rimon U, Salomon O, Halak M. Acute renal artery occlusion: presentation, treatment, and outcome. J Vasc Surg 2016;64(04):1026-1032

15 Paris B, Bobrie G, Rossignol P, Le Coz S, Chedid A, Plouin PF. Blood pressure and renal outcomes in patients with kidney infarction and hypertension. J Hypertens 2006;24(08):1649-1654 